\title{
Associated Factors and Liver Disease Severity for Decreased Bone Mineral Density in HIV Mono- and HIV/HCV Co-infected Patients
}

Valentina Li Vecchi' ${ }^{1}$, Maurizio Soresi' ${ }^{1}$, Lydia Giannitrapani' ${ }^{1}$, Giovanni Mazzola², Pietro Colletti², Ilaria Domenica Amico ${ }^{3}$, Fabio Tramuto ${ }^{3}$, Walter Granà', Massimo Midiri', Giuseppe Caruso ${ }^{4}$, Giuseppe Montalto ${ }^{*}$ and Paola Di Carlo ${ }^{3}$

${ }^{1}$ Biomedical Department of Internal Medicine and Specialties, University of Palermo, Italy

${ }^{2}$ Department of Clinical Medicine and Emerging Pathologies, University hospital P. Giaccone-Palermo, Italy

${ }^{3}$ Department of Sciences for Health Promotion and Mother-Child Care "G D'Alessandro", University of Palermo, Italy

${ }^{4}$ Department of Diagnostic Imaging, University of Palermo, Italy

Footnotes: Part of the study results has been presented as oral communication to the Italian Conference on AIDS and Retroviruses Roma, 25-27 May, 2014

\begin{abstract}
Objective: We assessed the prevalence and risk factors of decreased bone mineral density (BMD) in patients mono-infected with human immunodeficiency virus (HIV) or co-infected with hepatitis $\mathrm{C}$ virus (HIV/HCV). We also evaluated whether bone loss was linked to lipid asset in both groups and to severity of liver fibrosis in the co-infected group.
\end{abstract}

Methods: We consecutively enrolled 194 HIV-patients (129 mono-infected and 65 co-infected). All HIV-patients underwent dual-energy X-ray absorptiometry (DXA), while co-infected patients underwent transient elastography. Advanced liver fibrosis was defined as a median liver stiffness $\geq 9.5 \mathrm{kPa}$. Fibrosis was also assessed in all the HIVpatients using FIB-4.

Results: The overall prevalence of low BMD and osteoporosis was $26.8 \%$ and $26.0 \%$, respectively. It was significantly higher among HIV/HCV co-infected than mono-infected patients in lumbar/femoral sites $(P<0.04$ and $P<0.05$, respectively). HDL-cholesterol levels correlated independently with lumbar DXA Z-score $(P<0.03)$ in HIV mono-infected subjects. Liver stiffness correlated negatively and independently with femoral Z- and T-scores among co-infected patients $(P<0.003 ; P<0.01$, respectively). Stratifying co-infected subjects by sex, liver stiffness and lumbar/ femoral Z-scores $(P<0.04)$ or $T$-scores $(P<0.05 ; P<0.04$, respectively) correlated negatively only in the females. Longer $\mathrm{PI}$ exposure was negatively and independently correlated with BMD.

Conclusion: Our HIV-infected patients appeared at high risk for low BMD and osteoporosis. Severity of liver fibrosis was an independent predictor of bone loss in co-infection, although other factors could affect the skeletal system in HIV/HCV co-infection. Further research into the impact of liver fibrosis and lipid asset on bone disease in HIV-infection is necessary.

Keywords: HIV; HCV; Coinfection; Bone mineral density; Osteoporosis; Liver fibrosis

\section{Introduction}

Osteoporosis and fragility fractures are increasingly being recognized in people with human immunodeficiency virus (HIV) infection $[1,2]$. A meta-analysis of cross-sectional studies shows that the prevalence of osteoporosis in HIV-infected patients is approximately $15 \%$, which is more than three times greater than figures reported in HIV-uninfected controls [3].

In the last few years, many efforts have been made to identify the several and multifactorial mechanisms that may be involved in skeletal disorders in the HIV-setting [1,4].

Highly active antiretroviral therapy (HAART) has led to a significant reduction in morbidity and mortality in HIV-patients, although its long-term toxicity has been largely recognized $[3,5,6]$. The resulting aging of the HIV-population and a variety of effects related to specific antiretroviral drug exposure have been associated to the skeletal disorders $[3,7,8]$. A number of the traditional osteoporosis risk factors may be more prevalent in HIV-infected patients: vitamin D deficiency [9], low body weight $[7,10]$, lifestyle risk factors (including alcohol consumption [7], smoking, corticosteroid use [11] and drug addiction [12]), as well as the HIV infection per se (due to immunodeficiency). In addition, the chronic pro-inflammatory status and the direct action of HIV proteins may also play a role in decreasing bone mineral density (BMD) [4].
Hepatitis $\mathrm{C}$ virus (HCV) infection is a major health problem in the HIV-infected population [13]. A recent systematic review and meta-analysis suggests that $\mathrm{HIV} / \mathrm{HCV}$ co-infection is associated with a greater risk of osteoporosis than HIV mono-infection [14], although many divergences exist regarding osteoporosis prevalence in co-infected patients, with values ranging from $5-45 \%$. Moreover, it is debated whether liver disease severity may indeed have a relevant role in increasing the risk of osteoporosis in co-infection [15].

The relationship between lipid abnormalities and BMD has been little investigated in the setting of HIV-infection [16].

In this study we analyzed the prevalence of low BMD and frank osteoporosis in the whole HIV-population; we also studied both the

*Corresponding author: Giuseppe Montalto, Biomedical Department of Internal Medicine and Specialties, University of Palermo, Via del Vespro 141, Palermo, I-90127, Italy, Tel: +39-091 6552991; Fax+39-091 6552874; E-mail: giuseppe.montalto@unipa.it

Received November 07, 2014; Accepted March 02, 2015; Published March 10 2015

Citation: Li Vecchi V, Soresi M, Giannitrapani L, Mazzola G, Colletti P, et al. (2015) Associated Factors and Liver Disease Severity for Decreased Bone Mineral Density in HIV Mono- and HIV/HCV Co-infected Patients. J AIDS Clin Res 6: 438 doi:10.4172/2155-6113.1000438

Copyright: (c) 2015 Li Vecchi V, et al. This is an open-access article distributed under the terms of the Creative Commons Attribution License, which permits unrestricted use, distribution, and reproduction in any medium, provided the original author and source are credited. 
HIV/HCV co-infected and HIV mono-infected groups to investigate (i) what potential metabolic, lifestyle and/or HIV-related risk factors are associated with decreased BMD, and (ii) whether bone loss is more prevalent in HIV/HCV co-infected patients and if there is a link with severity of liver fibrosis.

\section{Methods}

\section{Study design}

This was a cross-sectional study, including consecutively enrolled HIV mono- and HIV/HCV co-infected patients who were being followed-up at the AIDS Center of the University of Palermo, Italy between January 2011 and May 2013.

Exclusion criteria included acute liver events, hepatocellular carcinoma, chronic hepatitis B infection, kidney disease, gastrointestinal disorders and steroid or sex steroid therapy.

The study protocol conformed to the ethical guidelines of the 1975 Declaration of Helsinki and was approved by the local Ethics Committee. Informed consent was obtained from all subjects.

\section{Data collection}

Data on age, gender and ethnicity, total time on antiretroviral therapy (ART), as well as exposure to protease inhibitors (PIs), nonnucleoside reverse-transcriptase inhibitors (NNRTIs) and tenofovir were collected, and recorded in a database designed for this study. Specific exposure $>1$ year to tenofovir and some other antiretroviral drugs (such as the first generation of HIV-1 integrase strand transfer inhibitors, raltegravir, or the NNRTIs, etravirine and PIs, darunavir) was also recorded. Exposure to PIs was graded in the following score (PI score): $0-3$ ( $0=$ no exposure; 1 : $<$ five years; 2 : from five to nine years; $3: \geq$ ten years).

HIV diagnosis date, medical and drug abuse history, lifestyle habits (including smoking status and alcohol consumption) and bone fractures were also recorded through patient interviews. Smoking status was categorized as non-smokers, former smokers and current smokers. Alcohol intake $>20 \mathrm{gm} /$ day either at the time of the study or in the past was also recorded.

Body mass index (BMI) was calculated as weight (in $\mathrm{kg}$ ) divided by height squared $\left(\mathrm{m}^{2}\right)$. Diabetes mellitus or Impaired Fasting Glucose (IFG) is defined in accordance with the Expert Committee on the Diagnosis and Classification of Diabetes Mellitus criteria [17]. Diabetes and IFG were analyzed together because of the small number of patients.

\section{Laboratory methods}

CD4+ T-cell count, and nadir, plasma HIV-RNA levels, hepatitis $B$ virus surface antigen and HCV antibody were assessed within two weeks after DXA. In the HIV/HCV co-infected patients HCV-genotype and plasma HCV-RNA levels were also recorded. Complete blood cell count, alanine aminotransferase (ALT) and aspartate aminotransferase (AST) were assessed within two weeks after DXA in the HIV mono- and HIV/HCV co-infected patients, respectively. Total-cholesterol, highdensity lipoprotein (HDL)-cholesterol, low-density lipoprotein (LDL)cholesterol, triglycerides and glycemia were determined at baseline in both the HIV-groups. The plasma concentration ratio of triglycerides to HDL (triglycerides/HDL ratio) was also calculated as a surrogate marker of insulin resistance [18]. Serum bone alkaline phosphatase, 25-hydroxyvitamin D, parathyroid hormone, phosphorus and calcium levels were assayed at the time of DXA scan in both the HIV-groups. All the blood tests were performed on samples from fasting patients.

\section{Bone mineral density assessment}

BMD was assessed in all patients by dual-energy X-ray absorptiometry (DXA), using a QDR Discovery Hologic DXA in the femoral neck and DXA in the lumbar spine by total body DXA, as previously reported [12]. For each scan, BMD, T-scores and Z-scores were recorded. T-score compares BMD with the mean of a healthy young (age 20-30 years) reference population, matched for sex and ethnicity, and is expressed as the number of standard deviations above or below the reference mean. Z-score is the number of standard deviations below the reference mean BMD of a gender- and ethnicitymatched population of the same age. Osteopenia was defined when at least one of the two DXA T-scores was less than -1. Osteoporosis was diagnosed when either femoral neck or lumbar spine DXA T-scores were less than -2.5 , as recommended by the World Health Organization (WHO) and the National Osteoporosis Foundation guidelines [19,20]. Low BMD was defined as a lumbar and/or femoral neck Z-score of -2 or less.

\section{Liver fibrosis assessment}

Transient elastography (FibroScan'; EchoSens, Paris, France), which assesses liver fibrosis by measuring liver stiffness, was performed in the $\mathrm{HIV} / \mathrm{HCV}$ co-infected patients. Details of the technical background and examination procedure have previously been described [21]. Liver cirrhosis was defined as liver stiffness $\geq 12.5 \mathrm{kPa}$ [22]. Fibrosis was assessed biologically using the FIB-4 index. It was calculated as follows: age $\times$ AST $[\mathrm{IU} / \mathrm{L}] /\left[\left(\right.\right.$ platelet count $\left.\left.\left[10^{9} / \mathrm{L}\right]\right) \times(\text { ALT }[\mathrm{IU} / \mathrm{L}])^{1 / 2}\right][23]$ Advanced liver fibrosis was estimated using an FIB-4 index $>3.25$ as a reference.

\section{Statistical analysis}

Data were expressed as mean \pm standard deviation when distribution was Gaussian. Differences were calculated using Student's t-test. Otherwise, data were expressed as median and range and analyzed with the Mann-Whitney $U$ test. Fisher's exact and $\chi^{2}$ tests, Pearson's correlation and Spearman's rank correlation were used where appropriate. Multiple linear regression analysis was performed to estimate the independence of the association between lumbar spine and femoral neck T-scores as well as variables significant at univariate analysis in all the HIV-infected subjects. Variables contributing significantly to fit the logistic equation were then selected by a step-wise procedure. $\mathrm{P}<0.05$ was considered significant.

All analyses were performed using the SPSS software package (version 22. 0; Chicago, IL, SA).

\section{Results}

\section{Study population}

From 260 elegible HIV-positive we enrolled 194 patients (121 M, 73 F), 129 of which were HIV mono-infected (66.5\%). Mean age was $48 \pm$ 8 years; 89/194 (45.9\%) patients were $>50$ years old.

Table 1 lists the main demographic, lifestyle and clinical features of the whole population and of the two study groups.

No significant differences were found in mean age or BMI values between the HIV mono-infected and co-infected patients. Analysis of life style risk factors showed that more than $50 \%$ of the study patients were smokers or ex-smokers $(\mathrm{P}<0.03)$. In addition, alcohol abuse $(\mathrm{P}<0.001)$ and drug addiction $(\mathrm{P}<0.0001)$ were significantly more prevalent in the co-infected $v s$. HIV mono-infected patients. 


\begin{tabular}{|c|c|c|c|c|}
\hline Characteristics & $\begin{array}{c}\text { Total } \\
n=194\end{array}$ & $\begin{array}{c}\text { HIV } \\
n=129\end{array}$ & $\begin{array}{c}\mathrm{HIV} / \mathrm{HCV} \\
n=65\end{array}$ & $P<$ \\
\hline Age years \pm SD & $48.3 \pm 8.5$ & $48.5 \pm 9.4$ & $47.7 \pm 5.9$ & ns \\
\hline Male/Female & $121 / 73$ & $74 / 55$ & $47 / 18$ & ns \\
\hline BMI $\left(\mathrm{Kg} / \mathrm{m}^{2}\right) \pm \mathrm{SD}$ & $23.5 \pm 3.1$ & $23.8 \pm 3.4$ & $22.8 \pm 2.7$ & ns \\
\hline Fracture $(n, \%)$ & $15(7.7)$ & $7(5.4)$ & $8(12.3)$ & ns \\
\hline $\begin{array}{c}\text { Ethnicity }(n, \%): \\
\text { Caucasian }\end{array}$ & $181(93.3)$ & $119(92.2)$ & $62(95.3)$ & ns \\
\hline African & $13(6.7)$ & $10(7.7)$ & $3(4.6)$ & ns \\
\hline $\begin{array}{l}\text { Smoking status }(n, \%): \\
\text { Non-smokers }\end{array}$ & $62(32.0)$ & $50(38.7)$ & $12(18.4)$ & \multirow[b]{3}{*}{0.03} \\
\hline Smokers & $97(50.0)$ & $57(44.2)$ & $40(61.5)$ & \\
\hline Former smokers & $35(28 \%)$ & $22(17)$ & $13(20)$ & \\
\hline Alcohol ( $n, \%)$ & $20(10.3)$ & $6(4.6)$ & $14(21.5)$ & 0.001 \\
\hline Drug addiction $(n, \%)$ & $56(28.8)$ & $14(10.8)$ & $42(64.6)$ & 0.0001 \\
\hline IFG/Diabetes ( $n, \%)$ & $45(23.2)$ & $28(21.7)$ & $17(26.1)$ & ns \\
\hline Time since HIV diagnosis (years) (range) & $15(0.1-29)$ & $12(0.1-28)$ & $18(0.1-29)$ & 0.0001 \\
\hline Treatment-naïve ( $n, \%)$ & $9(4.7)$ & $7(5.4)$ & $2(3.0)$ & ns \\
\hline Previous antiretroviral therapy (years) (range) & $9(0.5-26)$ & $8(0.5-23)$ & $13(0.5-26)$ & 0.0001 \\
\hline Genotype 1/others ( $n, \%)$ & - & - & $40(61)$ & - \\
\hline
\end{tabular}

HIV: Human Immunodeficiency Virus; HCV: Hepatitis C Virus; SD: Standard Deviation; BMI: Body Mass Index; IFG: Impaired Fasting Glucose; FRS: Framingham Risk Score; ns: Not Significant.

Table 1: Baseline demographic, lifestyle and clinical characteristics of the194 study patients.

\begin{tabular}{|c|c|c|c|c|}
\hline CHARACTERISTICS & $\begin{array}{l}\text { Total } \\
n=194\end{array}$ & $\begin{array}{c}\text { HIV } \\
n=29\end{array}$ & $\begin{array}{c}\mathrm{HIV} / \mathrm{HCV} \\
n=65\end{array}$ & $P<$ \\
\hline ALT U/L (range) & $28.0(10-175)$ & $24(10-171)$ & $43(13-175)$ & 0.0001 \\
\hline AST U/L (range) & $24.0(10-281)$ & $22(10-80)$ & $41(13-281)$ & 0.0001 \\
\hline Total-cholesterol $(\mathrm{mg} / \mathrm{dL}) \pm \mathrm{SD}$ & $195.2 \pm 51.7$ & $208.8 \pm 47.1$ & $165.7 \pm 49.9$ & 0.0001 \\
\hline HDL-cholesterol $(\mathrm{mg} / \mathrm{dL}) \pm \mathrm{SD}$ & $50.7 \pm 17.4$ & $51.7 \pm 14.8$ & $46.5 \pm 19.3$ & 0.05 \\
\hline LDL-cholesterol $(\mathrm{mg} / \mathrm{dL}) \pm \mathrm{SD}$ & $124.2 \pm 45.4$ & $133.5 \pm 42.8$ & $90.3 \pm 36.1$ & 0.0001 \\
\hline Triglicerides (mg/dL) (range) & $132(35-637)$ & $134(35-637)$ & $121.5(52-429)$ & ns \\
\hline Triglicerides/HDL ratio & $2.7(0.5-14)$ & $2.5(0.5-20)$ & $3.2(0.7-14)$ & ns \\
\hline Calcium (mg/dL) & $9.2 \pm 0.51$ & $9.1 \pm 0.6$ & $9.2 \pm 0.5$ & ns \\
\hline Phosphorus (mg/dL)* & $3.2 \pm 0.6$ & $3.2 \pm 0.6$ & $3.2 \pm 0.8$ & ns \\
\hline Parathyroid hormone $(\mathrm{pg} / \mathrm{mL})^{\circ}$ & $37.0(11-362)$ & $37.1(12-119)$ & $36(11-362)$ & ns \\
\hline Bone alkaline phosphatase (IU/L)ף & $46.5(17.2-170)$ & $46.8(17.2-116)$ & $46.2(17.4-170)$ & ns \\
\hline 25-Hydroxyvitamin $D$ (ng/mL) & $18.4(3-88.3)$ & $16.7(3-68)$ & $27.2(4.8-88.3)$ & 0.0001 \\
\hline HCV-RNA $(\mathrm{UI} / \mathrm{mL})>700.000(n, \%)$ & - & - & $41(63)$ & - \\
\hline CD4+ T-cell count (cells/ $\mu \mathrm{L})$ (range) & $480(34-1338)$ & $533.5(34-1082)$ & $413.5(10-1338)$ & 0.01 \\
\hline Nadir CD4+ T-cell count $<200$ (cells/ $\mu \mathrm{L})$ & $136(70.1)$ & $88(68)$ & $49(75)$ & ns \\
\hline Detectable HIV-RNA ( $n, \%)$ & $54(57.4)$ & $30(23.2)$ & $24(36.9)$ & ns \\
\hline
\end{tabular}

HIV: Human Immunodeficiency Virus; HCV: Hepatitis C Virus; ALT: Alanine Aminotransferase; AST: Aspartate Aminotransferase; HDL: High-Density Lipoprotein; LDL: LowDensity Lipoprotein; ns: Not Significant. ${ }^{*}$ Missing in $10 \mathrm{HIV}$ mono-infected and 2 HIV/HCV co-infected patients; ${ }^{\circ}$ Missing in 6 HIV mono-infected and 3 HIV/HCV co-infected patients; Missing in 5 HIV mono-infected patients.

Table 2: Main biological parameters.

Most HIV mono- and HIV/HCV co-infected patients (95\%) were following HAART: 9 patients (7 HIV mono- and 2 HIV/HCV co-infected) were treatment-naïve, while only 1 HIV mono-infected patient had interrupted treatment due to poor compliance. Time since HIV diagnosis and duration of ART exposure were both significantly longer in the co-infected than HIV mono-infected patients $(\mathrm{P}<0.0001)$.

Numerous HIV mono- and co-infected patients (46/121 vs. 34/63 patients) had received PIs for more than 10 years, although no significant difference in duration of PI exposure was observed between the two groups when the PI treatment score was calculated $(P=n s)$. Moreover, no significant difference was found either in the prevalence of exposure to tenofovir or to the newer antiretroviral drugs (data not shown).
Table 2 shows the main hematological and virological parameters in the study population. All of the HCV co-infected patients had detectable HCV-RNA and 41 patients (63\%) had HCV-RNA >700 000 $\mathrm{IU} / \mathrm{ml}$. Serum ALT and AST levels were significantly higher in coinfected than in mono-infected patients $(\mathrm{P}<0.0001)$. Total-cholesterol, LDL- and HDL-cholesterol levels were higher in the mono-infected than in co-infected patients $(\mathrm{P}<0.0001 ; \mathrm{P}<0.05$, respectively). The median level of vitamin D was lower in HIV mono-infected than HIV/ HCV co-infected patients $(\mathrm{P}<0.0001)$. CD4+T-cell count $($ cells $/ \mu \mathrm{l})$ median values were lower in patients with co-infection than in those with mono-infection $(\mathrm{P}<0.01)$.

Liver stiffness $\geq 12.5 \mathrm{kPa}$ was found in $27.6 \%$ of $\mathrm{HIV} / \mathrm{HCV}$ coinfected cases, with a higher percentage among the males [14/47 (29.7\%) males vs. 4/16 (25\%) females]. In $2 \mathrm{HIV} / \mathrm{HCV}$ co-infected females liver 
stiffness assessment was not performed because of decompensated liver disease.

\section{Prevalence of low bone mineral density/osteopenia/ osteoporosis}

135/194 study patients (69.5\%) had BMD values lower than the reference values for a healthy Caucasian population. In detail, significant skeletal disease, i.e. low BMD and frank osteoporosis, was found in $52(26.8 \%)$ and $50(26 \%)$ HIV-infected patients, respectively. Comparison between the two groups showed that overall prevalence of low BMD and osteoporosis in both the lumbar and femoral sites was significantly higher in HIV/HCV co-infected than in mono-infected patients ( $\mathrm{P}<0.04$ and $\mathrm{P}<0.05$, respectively) (Table 3 ).

As regards the analysis of BMD in the single sites, although lumbar and femoral DXA Z- and T-scores were significantly lower in $\mathrm{HIV} /$ HCV co-infected than in mono-infected subjects $(\mathrm{P}<0.02 ; \mathrm{P}<0.05)$, only in the lumbar site was the prevalence of low BMD and osteoporosis higher in co-infected vs. mono-infected subjects $(\mathrm{P}<0.03$ and $\mathrm{P}<0.02$, respectively) (Table 3 ).

\section{Correlation between BMD and extent of liver fibrosis}

In the HIV mono-infected group no significant correlations were observed between BMD measured with either lumbar or femoral DXA Z- and T-scores and liver fibrosis evaluated by FIB-4. In co-infected patients FIB-4 values negatively correlated either with lumbar and femoral DXA Z-scores ( $\mathrm{r}=-0.32$ and $-0.33 ; \mathrm{P}<0.01$, respectively) or lumbar and femoral DXA T-scores $(r=-0.3 ; \mathrm{P}<0.03$ and $\mathrm{r}=-0.31 ; \mathrm{P}<0.01$, respectively).

When HIV/HCV co-infected patients were stratified by sex, a significant and negative correlation between extent of liver stiffness and both the lumbar and femoral DXA Z-scores $[\mathrm{r}=-0.5 ;(\mathrm{P}<0.04)]$ or lumbar and femoral neck DXA T-scores $[\mathrm{r}=-0.45(\mathrm{P}<0.05) ; \mathrm{r}=-0.54$ $(\mathrm{P}<0.04)$, respectively] was found only in the female group (Figures 1 and 2).

\begin{tabular}{|c|c|c|c|}
\hline & $\begin{array}{c}\text { HIV+ } \\
n=129\end{array}$ & $\begin{array}{c}\mathrm{HIV} / \mathrm{HCV}+ \\
n=65\end{array}$ & $\mathrm{P}<$ \\
\hline \multicolumn{4}{|l|}{ BONE MINERAL DENSITY } \\
\hline \multicolumn{4}{|l|}{$\begin{array}{l}\text { Overall (femoral neck or } \\
\text { lumbar spine) }\end{array}$} \\
\hline Osteopenia $(n, \%)$ & $58(44.9)$ & $27(41.5)$ & ns \\
\hline Osteoporosis $(n, \%)$ & $27(20.9)$ & $23(33.8)$ & 0.05 \\
\hline Low BMD $(n, \%)$ & $28(21.7)$ & $24(36.9)$ & 0.04 \\
\hline \multicolumn{4}{|l|}{ Femoral neck } \\
\hline DXA Z-score \pm SD & $-0.3(-2.5-2.2)$ & $-0.8(-3.3-1.7)$ & 0.02 \\
\hline Low BMD $(n, \%)$ & $8(6.2)$ & $7(10.7)$ & ns \\
\hline DXA T-score & $-0.6(-2.8-1.8)$ & $-1(-3.6-1.4)$ & 0.02 \\
\hline Osteopenia $(n, \%)$ & $47(36.4)$ & $24(36.9)$ & ns \\
\hline Osteoporosis $(n, \%)$ & $3(2.3)$ & $5(7.7)$ & ns \\
\hline \multicolumn{4}{|l|}{ Lumbar spine } \\
\hline DXA Z-score & $-0.9(-3.7-2.7)$ & $-1.3(-4.5-1.3)$ & 0.02 \\
\hline Low BMD $(n, \%)$ & $27(20.9)$ & $24(36.9)$ & 0.03 \\
\hline DXA T-score \pm SD & $-1.3(-3.9-2.3)$ & $-1.7(-5.2-1)$ & 0.05 \\
\hline Osteopenia $(n, \%)$ & $50(38.7)$ & $24(36.9)$ & ns \\
\hline Osteoporosis $(n, \%)$ & $26(20)$ & $23(35.3)$ & 0.02 \\
\hline
\end{tabular}

HIV: Human Immunodeficiency Virus; HCV: Hepatitis C Virus; BMD: Bone Mineral Density; DXA: Dual-Energy X-Ray Absorptiometry.

Table 3: Bone mineral density measurements of the 194 study patients.

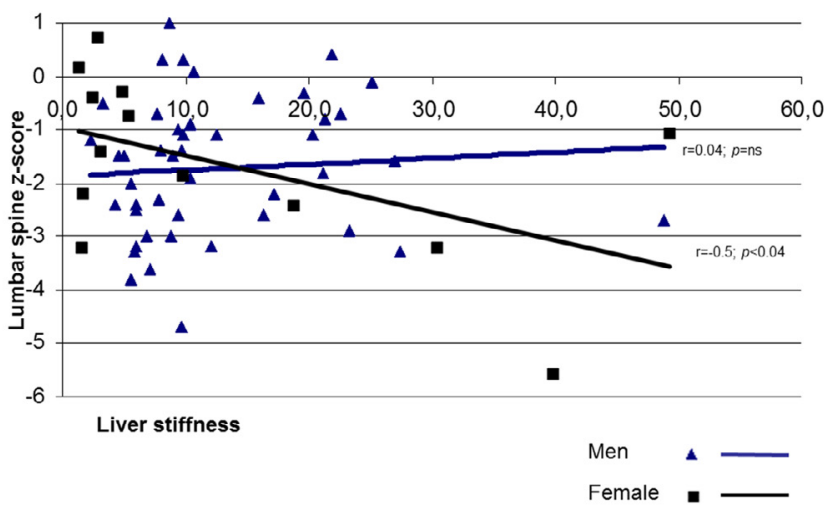

Figure 1: Shows the correlation between liver fibrosis, measured as liver stiffness and lumbar spine DXA Z-score in HIV/HCV co-infected patients divided according to sex.

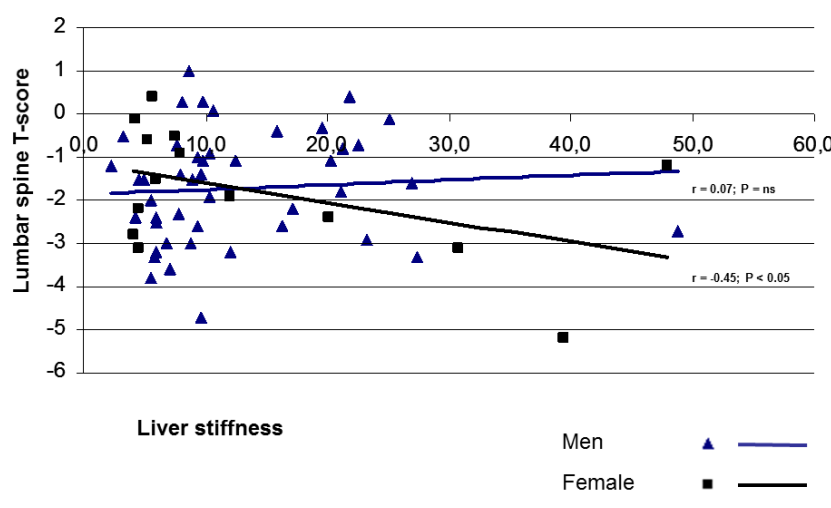

Figure 2: Shows the correlation between liver fibrosis, measured as liver stiffness and lumbar spine DXA T-score in HIV/HCV co-infected patients divided according to sex.

Figures 1 and 2 show the correlation between liver fibrosis, measured by transient elastography and lumbar spine DXA Z- and T-scores, respectively, in $\mathrm{HIV} / \mathrm{HCV}$ co-infected patients divided according to sex.

\section{Correlation between BMD and other study parameters in both the HIV-groups}

Tables 4 and 5 show the correlations between lumbar/femoral DXA Z- and T-scores and the variables analyzed in the two groups by univariate and multivariate analysis.

HIV mono-infected group: BMD negatively correlated with nadir CD4+ $<200($ cells/ $\mu$ l) in lumbar/femoral DXA Z-scores $(\mathrm{P}<0.05$; $\mathrm{P}<0.01$, respectively). Bone alkaline phosphatase $(\mathrm{P}<0.01)$ and $\mathrm{PI}$ score $(\mathrm{P}<0.02)$ negatively correlated with femoral DXA Z-score (Table 4$)$.

Lumbar/femoral DXA T-scores negatively correlated with age $(\mathrm{P}<0.01)$, bone alkaline phosphatase $(\mathrm{P}<0.01 ; \mathrm{P}<0.02$, respectively), nadir $\mathrm{CD} 4+<200$ (cells/ $\mu \mathrm{l})(\mathrm{P}<0.03 ; \mathrm{P}<0.01$, respectively), and $\mathrm{PI}$ score $(\mathrm{P}<0.02 ; \mathrm{P}<0.01$, respectively) (Table 5$)$. In addition, both $\mathrm{BMI}$ and HDL-cholesterol levels positively correlated with lumbar DXA Z-score $(\mathrm{P}<0.01 ; \mathrm{P}<0.03)$ (Table 4). A positive correlation was also found between BMI and femoral DXA Z-score $(\mathrm{P}<0.0001)$ (Table 4$)$.

HIV/HCV co-infected group: Bone alkaline phosphatase levels negatively correlated with lumbar/femoral DXA Z-scores $(\mathrm{P}<0.05$; 


\begin{tabular}{|c|c|c|c|c|c|c|c|c|c|c|c|c|c|c|c|c|}
\hline & \multicolumn{8}{|c|}{ Lumbar spine Z-score } & \multicolumn{8}{|c|}{ Femoral neck Z-score } \\
\hline & \multicolumn{4}{|c|}{ HIV mono-infected $n=129$} & \multicolumn{4}{|c|}{ HIV/HCV co-infected $n=65$} & \multicolumn{4}{|c|}{ HIV mono-infected $n=129$} & \multicolumn{4}{|c|}{ HIV/HCV co-infected $n=65$} \\
\hline & \multicolumn{4}{|c|}{ Analysis } & \multicolumn{4}{|c|}{ Analysis } & \multicolumn{4}{|c|}{ Analysis } & \multicolumn{4}{|c|}{ Analysis } \\
\hline & \multicolumn{2}{|c|}{ Univariate } & \multicolumn{2}{|c|}{ Multivariate } & \multicolumn{2}{|c|}{ Univariate } & \multicolumn{2}{|c|}{ Multivariate } & \multicolumn{2}{|c|}{ Univariate } & \multicolumn{2}{|c|}{ Multivariate } & \multicolumn{2}{|c|}{ Univariate } & \multicolumn{2}{|c|}{ Multivariate } \\
\hline $\begin{array}{l}\text { Demographic/lifestyle } \\
\text { characteristics }\end{array}$ & & $\mathrm{P}<$ & $\beta$ & $\mathrm{P}<$ & & $\mathrm{P}<$ & $\beta$ & $\mathrm{P}<$ & & $\mathrm{P}<$ & $\beta$ & $\mathrm{P}<$ & & $\mathrm{P}<$ & $\beta$ & $\mathrm{P}<$ \\
\hline Age & $0.03^{*}$ & ns & - & - & $-0.12^{*}$ & ns & - & - & $-0.18^{*}$ & ns & - & - & -0.17 & ns & - & - \\
\hline BMI $\left(\mathrm{kg} / \mathrm{m}^{2}\right)$ & $0.25^{*}$ & 0.01 & 0.30 & 0.01 & $0.05^{*}$ & ns & - & - & $0.44^{*}$ & 0.0001 & 0.45 & 0.0001 & $0.26^{*}$ & 0.05 & 0.33 & 0.02 \\
\hline Menopause & $0.02^{\circ}$ & ns & - & - & $-0.02^{\circ}$ & ns & - & - & $-0.06^{\circ}$ & ns & - & - & $-0.18^{\circ}$ & ns & - & - \\
\hline \multicolumn{17}{|l|}{ Biochemical parameters } \\
\hline $\begin{array}{l}\text { Bone alkaline phosphatase } \\
\text { (IU/L) }\end{array}$ & $-0.01^{*}$ & ns & - & - & $-0.25^{\star}$ & 0.05 & - & - & $-0.25^{*}$ & 0.01 & - & - & $-0.31^{*}$ & 0.02 & - & - \\
\hline HDL-cholesterol (mg/dL ) & $0.2^{*}$ & 0.03 & 0.22 & 0.02 & $0.09^{*}$ & ns & - & - & 0.01 & ns & - & - & $0.12^{*}$ & ns & - & - \\
\hline \multicolumn{17}{|l|}{ Characteristics of HIV infection } \\
\hline $\begin{array}{l}\text { Nadir CD4+<200 } \\
\text { (cells } / \mu \mathrm{L})\end{array}$ & $-0.21^{\circ}$ & 0.05 & - & - & $-0.03^{\circ}$ & ns & - & - & $-0.28^{\circ}$ & 0.01 & - & - & $-0.17^{\circ}$ & ns & - & - \\
\hline $\begin{array}{l}\text { Time since HIV } \\
\text { diagnosis }\end{array}$ & $-0.03^{*}$ & ns & - & - & $-0.2^{*}$ & ns & - & - & $-0.02^{*}$ & ns & - & - & $-0.04^{*}$ & ns & - & - \\
\hline Score of PI exposure & $-0.09^{\circ}$ & ns & - & - & $-0.19^{\circ}$ & ns & - & - & $-0.2^{\circ}$ & 0.02 & - & - & $-0.27^{\circ}$ & 0.04 & -0.32 & 0.02 \\
\hline
\end{tabular}

HIV: Human Immunodeficiency Virus; HCV: Hepatitis C Virus; DXA: Dual-Energy X-Ray Absorptiometry; BMI: Body Mass Index; Ns: Not Significant; HDL: High-Density Lipoprotein; PI: Protease Inhibitor. *R: Pearson's Correlation Test; ${ }^{\circ}$ Spearman's Rank Correlation Test.

Table 4: Correlation between lumbar spine, femoral neck DXA Z-scores and the study parameters.

\begin{tabular}{|c|c|c|c|c|c|c|c|c|c|c|c|c|c|c|c|c|}
\hline & \multicolumn{8}{|c|}{ Lumbar Spine T-score } & \multicolumn{8}{|c|}{ Femoral neck T-score } \\
\hline & \multirow{2}{*}{\multicolumn{4}{|c|}{$\begin{array}{l}\text { HIV mono-infected } n=129 \\
\text { Analysis }\end{array}$}} & \multicolumn{4}{|c|}{ HIV/HCV co-infected $n=65$} & \multicolumn{4}{|c|}{ HIV mono-infected $n=129$} & \multicolumn{4}{|c|}{ HIV/HCV co-infected $n=65$} \\
\hline \multirow[b]{3}{*}{$\begin{array}{l}\text { Demographic/lifestyle } \\
\text { characteristics }\end{array}$} & & & & & & Ane & sis & & & Ans & /sis & & & Ana & ysis & \\
\hline & \multicolumn{2}{|c|}{ Univariate } & \multicolumn{2}{|c|}{ Multivariate } & \multicolumn{2}{|c|}{ Univariate } & \multicolumn{2}{|c|}{ Multivariate } & \multicolumn{2}{|c|}{ Univariate } & \multicolumn{2}{|c|}{ Multivariate } & \multicolumn{2}{|c|}{ Univariate } & \multicolumn{2}{|c|}{ Multivariate } \\
\hline & & $\mathrm{P}<$ & $\beta$ & $\mathrm{P}<$ & & $\mathrm{P}<$ & $\beta$ & $\mathrm{P}<$ & & $\mathrm{P}<$ & $\beta$ & $\mathrm{P}<$ & & $\mathrm{P}<$ & $\beta$ & $\mathrm{P}<$ \\
\hline Age & $-0.24^{*}$ & 0.01 & - & - & -0.16 & ns & - & - & -0.22 & 0.01 & - & - & $-0.18^{*}$ & ns & - & - \\
\hline $\operatorname{BMI}\left(\mathrm{kg} / \mathrm{m}^{2}\right)$ & $0.17^{*}$ & Ns & - & - & $0.15^{*}$ & ns & - & - & $0.25^{*}$ & 0.01 & - & - & $0.27^{*}$ & 0.04 & 0.36 & 0.03 \\
\hline Menopause & $-0.04^{\circ}$ & ns & - & - & $-0.3^{\circ}$ & 0.02 & - & - & $-0.03^{\circ}$ & ns & - & - & $-0.27^{\circ}$ & 0.04 & - & - \\
\hline \multicolumn{17}{|l|}{ Biochemical parameters } \\
\hline $\begin{array}{l}\text { Bone alkaline phosphatase } \\
\text { (IU/L) }\end{array}$ & $-0.3^{*}$ & 0.01 & - & - & $-0.26^{*}$ & 0.05 & - & - & $-0.28^{*}$ & 0.02 & - & - & $-0.34^{*}$ & 0.02 & - & - \\
\hline HDL-cholesterol (mg/dL ) & $0.08^{*}$ & ns & - & - & $0.07^{*}$ & ns & - & - & $0.04^{*}$ & ns & - & - & $0.11^{*}$ & ns & - & - \\
\hline \multicolumn{17}{|l|}{ Characteristics of HIV infection } \\
\hline $\begin{array}{l}\text { Nadir CD4+ }<200 \\
(\text { cells } / \mu \mathrm{L})\end{array}$ & $-0.22^{\circ}$ & 0.03 & - & - & $-0.17^{\circ}$ & ns & - & - & $-0.28^{\circ}$ & 0.01 & - & - & $-0.17^{\circ}$ & ns & - & - \\
\hline $\begin{array}{l}\text { Time since HIV } \\
\text { diagnosis }\end{array}$ & $-0.20^{*}$ & 0.03 & - & - & $-0.14^{*}$ & ns & - & - & $-0.14^{*}$ & ns & - & - & $-0.05^{*}$ & ns & - & - \\
\hline Score of PI exposure & $-0.2^{\circ}$ & 0.02 & -0.33 & 0.02 & $-0.25^{\circ}$ & 0.05 & - & - & $-0.25^{\circ}$ & 0.01 & -0.5 & 0.0001 & $-0.4^{\circ}$ & 0.02 & -0.32 & 0.02 \\
\hline
\end{tabular}

HIV: Human Immunodeficiency Virus; HCV: Hepatitis C Virus; DXA: Dual-Energy X-Ray Absorptiometry; BMI: Body Mass Index; Ns: Not Significant; HDL: High-Density Lipoprotein; PI: Protease Inhibitor. *R: Pearson's Correlation Test; 'Spearman's Rank Correlation Test.

Table 5: Correlation between lumbar spine, femoral neck DXA T-scores and the study parameters.

$\mathrm{P}<0.02$, respectively). Femoral DXA Z-score negatively correlated with the PI score $(\mathrm{P}<0.04)$ (Table 4$)$.

Menopause, as well as bone alkaline phosphatase and PI score negatively correlated with lumbar/femoral DXA T-scores $(\mathrm{P}<0.02$, $\mathrm{P}<0.05 ; \mathrm{P}<0.04, \mathrm{P}<0.02$, respectively) (Table 5).

A positive correlation was found only between BMI and femoral DXA T-score $(\mathrm{P}<0.04)$.

In the whole HIV-population a significant correlation was found between low BMD and HIV/HCV co-infection (rho: $0.2 ; \mathrm{P}<0.02$ ), totalcholesterol (rho: $-0.2 ; \mathrm{P}<0.02$ ) as well as FIB-4 score (rho 0.15; $\mathrm{P}<0.05$ ). In the HIV mono-infected group low BMD was correlated with totalcholesterol (rho $-0.2 ; \mathrm{P}<0.05$ ) and ART $>5$ years (rho $-0.2 ; \mathrm{P}<0.05$ ).

Among the variables significant at univariate analysis, low BMD was independently correlated with FIB-4 OR: 1.3 (95 CI 1.1-1.6) $\mathrm{P}<0.02$. No significant independent correlation was found between low $\mathrm{BMD}$ and $\mathrm{HIV} / \mathrm{HCV}$ co-infection.

On multiple linear regression analysis an independent and positive correlation was found between BMI and DXA Z-score measured in both sites (lumbar spine and femoral neck) in HIV mono-infected patients $(\beta=0.30 ; \mathrm{P}<0.01 ; \beta=0.45 ; \mathrm{P}<0.0001$, respectively). Lumbar spine DXA Z-score was also independently correlated with HDLcholesterol $(\beta=0.22 ; \mathrm{P}<0.02)$ (Table 4). Lumbar /femoral DXA T-scores were negatively and independently correlated with PI use score in the HIV mono-infected group $(\beta=-0.33 ; \mathrm{P}<0.02 ; \beta=-0.50 ; \mathrm{P}<0.0001$, respectively) (Table 5).

In $\mathrm{HIV} / \mathrm{HCV}$ co-infected patients an independent and positive correlation was found between BMI and both femoral DXA Z- and 
T-scores $(\beta=0.33 ; \mathrm{P}<0.02 ; \beta=0.36 ; \mathrm{P}<0.03$, respectively) (Tables 4,5 ); in the same group PI score negatively correlated with femoral DXA Z-score $(\beta=-0.32 ; \mathrm{P}<0.02)$ (Table 4$)$. Extent of liver stiffness was an independent predictor of bone loss in $\mathrm{HIV} / \mathrm{HCV}$ co-infected patients in the femoral DXA Z- and T-scores $(\beta=-0.43 ; \mathrm{P}<0.003 ; \beta=-0.41$; $\mathrm{P}<0.01$, respectively). FIB-4 values were negatively and independently correlated with lumbar DXA Z- and T-scores in HIV/HCV co-infected patients $(\beta=-0.43 ; \mathrm{P}<0.004 ; \beta=-0.46 ; \mathrm{P}<0.002$, respectively).

\section{Discussion}

In this cross-sectional study we found a very high prevalence of low BMD and osteoporosis among predominately Caucasian HIVinfected patients with or without chronic hepatitis $\mathrm{C}$, with long-term HIV-infection and prolonged ART. The overall $26 \%$ prevalence of frank osteoporosis in our HIV-cohort was higher than the percentages reported in a previous meta-analysis and in other European case studies $[1,3,24]$. In our HIV/HCV co-infected patients osteoporosis prevalence was significantly higher than among HIV mono-infected subjects, in agreement with previous findings [25]. Many divergences persist regarding the prevalence of osteoporosis in HIV/HCV co-infection [14]. The $33.8 \%$ osteoporosis prevalence in our HIV/HCV co-infected patients was higher than the pooled estimate of $22 \%$ taken from a recent meta-analysis [14]. Likewise, the prevalence of low BMD in our co-infected patients was higher than the result reported by El-Maouche et al. [15] (36.9\% vs. 26\%, respectively), as well as being considerably higher than the $16 \%$ observed in a large cohort of HIV/HCV co-infected patients from Modena (northern Italy) [26]. Although the proportion of enrolled co-infected males and females and sample size in the two Italian HIV cohorts were similar, other co-morbidities, as well as the heterogeneity of liver disease may account for the discrepancy in the low BMD prevalences.

Although controversial, a direct correlation between extent of liver fibrosis and low bone density has been reported in non-cirrhotic HCV mono-infected patients $[27,28]$. No association between liver disease severity and BMD at any site was reported in co-infected patients by ElMaouche et al. [15], despite the high histological evidence of cirrhosis (26\%). Liver cirrhosis, assessed by liver biopsy or non-invasive methods (FibroScan and FibroTest), was also not found to be associated with decreased BMD in co-infected patients by Lawson-Ayayi et al. [25]. In our study the extent of liver fibrosis, measured by transient elastography, was an independent predictor of bone loss in HIV/HCV co-infected patients in the femoral neck DXA Z- and T-scores. Of clinical interest, a significant negative correlation between BMD in both the lumbar spine/femoral neck sites and severity of hepatic fibrosis was found in our HIV/HCV co-infected females, but not males, suggesting that viral hepatitis co-infected women are even at risk for vertebral and femoral fractures. Although we did not assay sexual hormones, the correlation between menopause and lumbar/femoral DXA Z- and T-scores on univariate analysis suggests a role of estrogen deficiency for bone loss in our HIV/HCV co-infected women. This hypothesis is furthermore corroborated by the evidence of liver stiffness $\geq 12.5 \mathrm{kPa}$ in a higher percentage of co-infected males than females $(29.7 \%$ vs. $25 \%$, respectively), which in addition to the lack of a significant correlation between the extent of liver fibrosis and BMD in the males of the same co-infected group, suggests that bone abnormalities are more likely to be the result of HIV/HCV co-infection and female gender. Among the multifactorial mechanisms underlying skeletal disorder in the HIV and HCV setting, vitamin D deficiency is considered a risk factor for osteoporotic fracture [14]. Vitamin D levels were found to be lower in our HIV mono-infected patients than in subjects with HIV/HCV co- infections. In addition, the median vitamin $\mathrm{D}$ level was $>30 \mathrm{ng} / \mathrm{mL}$ in $\mathrm{HIV} / \mathrm{HCV}$ co-infected patients. Our results reflect those reported in an Italian study, which found that median vitamin D serum levels $>25 \mathrm{ng} /$ $\mathrm{ml}$ were similar in the HIV mono-infected, HIV/HCV co-infected and healthy controls [29]. Moreover, in a recent, large study on a general healthy population in Central Europe, Pludowski et al. [30] reported an average vitamin D concentration of $>30 \mathrm{ng} / \mathrm{ml}$. However, since the Hormone Foundation's Patient Guide to Vitamin D Deficiency suggests that patients with chronic (long-term) liver disease are at high risk of deficiency [31], further randomized controlled trials are warranted to determine what level of vitamin D insufficiency and deficiency places an individual at risk for cirrhosis evolution.

The mechanisms underlying osteoporosis in HIV-infected patients with viral hepatitis have not been completely understood to date. The evidence gathered suggests a close link between bone disease and a state of chronic inflammation [4]. In this respect, the HIV-related immune activation induces cytokine changes that may increase liver inflammation and fibrosis, which in turn may result in an increase in osteoclastic activity, mediated by the osteoclastogenic pro-inflammatory cytokines [4,32,33]. HIV-associated chronic inflammation may also cause endothelial dysfunction, atheroma formation and acute thrombosis, which are determinants of cardiovascular diseases in HIV-infection [34], and this may represent a potential link between bone disease, liver disease and cardiovascular risk in HIV/HCV coinfection. Since cardiovascular risk has been negatively associated to low HDL-cholesterol levels, a relationship between HDL-cholesterol and BMD has been postulated [35]. Our data showed an independent and positive correlation between lumbar spine DXA Z-score and HDLcholesterol levels in HIV mono-infected patients. The link between lipid abnormalities and low BMD has been little investigated in the HIV-setting [16,36].

Several HIV-related factors negatively correlated with BMD in the lumbar/femoral DXA Z- and T- scores of our mono-infected and coinfected patients on univariate analysis. The correlation between time of HIV diagnosis, as well as nadir CD4+ T-cell count $<200$ cells/ $\mu \mathrm{l}$ and BMD suggest that HIV infection per se and in particular the more advanced stages of the disease might affect the skeletal system. The negative and independent correlation between longer PI exposure and BMD supports earlier evidence on the unfavorable role of PI exposure in the progression of bone loss [12].

Low BMI values were independently correlated with low BMD. These findings support the report in a meta-analysis of 10 studies on HIV-infected patients that low body weight may largely account for the high prevalence of low BMD in the setting of HIV-infection [10].

Some limitations of the present study need to be mentioned. The small size and homogeneity of the sample do not allow a generalization of our results. In addition, the cross-sectional study design does not permit us to establish a cause-effect relationship in our results or to evaluate the impact of liver disease progression on bone metabolism. Our analysis is also limited by the lack of sexual hormone assessment and of the absence of markers of systemic inflammation, which would be useful in evaluating the impact of female sex or inflammation on bone health. Finally, the numerous variables evaluated are potentially confounding and the multivariate analyses may not entirely eliminate the residual confounding factors.

In conclusion, our Caucasian HIV mono- and HIV/HCV coinfected patients appeared at high risk for low BMD and osteoporosis. The extent of liver fibrosis, measured by transient elastography, was 
an independent predictor of bone loss in $\mathrm{HIV} / \mathrm{HCV}$ co-infection. When patients were stratified by sex, a negative correlation between $\mathrm{BMD}$ and severity of liver fibrosis was found in co-infected women only, suggesting that other factors (i.e. sexual hormones) may affect the skeletal system in HIV/HCV co-infection more than liver fibrosis. Additional research into the impact of chronic hepatitis $\mathrm{C}$ on osteoporosis and the interaction between lipid asset, cardiovascular risk and bone disease effects are required in the HIV infection setting.

\section{Acknowledgements} text.

We would like to thank Dr. Carole Greenall, BA, for the revision of the English

\section{References}

1. Bonjoch A, Figueras M, Estany C, Perez-Alvarez N, Rosales J, et al. (2010) High prevalence of and progression to low bone mineral density in HIV-infected patients: a longitudinal cohort study. AIDS 24: 2827-2833.

2. Triant VA, Brown TT, Lee H, Grinspoon SK (2008) Fracture prevalence among human immunodeficiency virus (HIV)-infected versus non-HIV-infected patients in a large U.S. healthcare system. J Clin Endocrinol Metab 93: 3499-3504.

3. Brown TT, Qaqish RB (2006) Antiretroviral therapy and the prevalence of osteopenia and osteoporosis: a meta-analytic review. AIDS 20: 2165-2174.

4. Ofotokun I, Mclntosh E, Weitzmann MN (2012) HIV: inflammation and bone. Curr HIVIAIDS Rep 9: 16-25.

5. Palella FJ Jr, Delaney KM, Moorman AC, Loveless MO, Fuhrer J, et al. (1998) Declining morbidity and mortality among patients with advanced human immunodeficiency virus infection. HIV Outpatient Study Investigators. N Engl J Med 338: 853-860.

6. Grinspoon S, Carr A (2005) Cardiovascular risk and body-fat abnormalities in HIV-infected adults.N Engl J Med 352: 48-62.

7. Mondy K, Yarasheski K, Powderly WG, Whyte M, Claxton S, et al. (2003) Longitudinal evolution of bone mineral density and bone markers in human immunodeficiency virus-infected individuals. Clin Infect Dis 36: 482-490.

8. Arnsten JH, Freeman R, Howard AA, Floris-Moore M, Lo Y, Klein RS (2007) Decreased bone mineral density and increased fracture risk in aging men with or at risk for HIV infection. AIDS 21: 617-623.

9. Dao CN, Patel P, Overton ET, Rhame F, Pals SL, et al. (2011) Low vitamin D among HIV-infected adults: prevalence of and risk factors for low vitamin D Levels in a cohort of HIV-infected adults and comparison to prevalence among adults in the US general population. Clin Infect Dis 52: 396-405.

10. Bolland MJ, Grey AB, Gamble GD, Reid IR (2007) CLINICAL Review \#: low body weight mediates the relationship between HIV infection and low bone mineral density: a meta-analysis. J Clin Endocrinol Metab 92: 4522-4528.

11. Womack JA, Goulet JL, Gibert C, Brandt C, Chang CC, et al. (2011) Increased risk of fragility fractures among HIV infected compared to uninfected male veterans. PLoS One 6: e17217.

12. Li Vecchi V, Soresi M, Giannitrapani L, Mazzola G, La Sala S, et al. (2012) Dairy calcium intake and lifestyle risk factors for bone loss in HIV-infected and uninfected Mediterranean subjects. BMC Infect Dis 12: 192

13. Machado MV, Oliveira AG, Cortez-Pinto H (2010) Hepatic steatosis in patients coinfected with human immunodeficiency virus/hepatitis $C$ virus: a metaanalysis of the risk factors. Hepatology 52: 71-78.

14. Dong HV, Cortés YI, Shiau S, Yin MT (2014) Osteoporosis and fractures in HIV/ hepatitis $C$ virus coinfection: a systematic review and meta-analysis. AIDS 28 : 2119-2131.

15. El-Maouche D, Mehta SH, Sutcliffe C, Higgins Y, Torbenson MS, et al. (2011) Controlled HIV viral replication, not liver disease severity associated with low bone mineral density in HIV/HCV co-infection. J Hepatol 55: 770-776.

16. Anuurad E, Semrad A, Berglund L (2009) Human immunodeficiency virus and highly active antiretroviral therapy-associated metabolic disorders and risk factors for cardiovascular disease. Metab Syndr Relat Disord 7: 401-410.

17. Alberti KG, Zimmet P, Shaw J; IDF Epidemiology Task Force Consensus Group (2005) The metabolic syndrome--a new worldwide definition. Lancet 366: 10591062.
18. Sung KC, Ryan MC, Kim BS, Cho YK, Kim BI, et al. (2007) Relationships between estimates of adiposity, insulin resistance, and nonalcoholic fatty liver disease in a large group of nondiabetic Korean adults. Diabetes Care 30: 2113 2118.

19. (1994) Assessment of fracture risk and its application to screening for postmenopausal osteoporosis. Report of a WHO Study Group. World Health Organ Tech Rep Ser 843: 1-129.

20. National Osteoporosis Foundation (2010) Clinician's Guide to Prevention and Treatment of Osteoporosis. National Osteoporosis Foundation, Washington, DC.

21. Li Vecchi V, Soresi M, Colomba C, Mazzola G, Colletti P, et al. (2010) Transient elastography: a non-invasive tool for assessing liver fibrosis in HIV/HCV patients. World J Gastroenterol 16: 5225-5232.

22. Castéra L, Vergniol J, Foucher J, Le Bail B, Chanteloup E, et al. (2005) Prospective comparison of transient elastography, Fibrotest, APRI, and liver biopsy for the assessment of fibrosis in chronic hepatitis C. Gastroenterology 128: $343-350$.

23. Sterling RK, Lissen E, Clumeck N, Sola R, Correa MC, et al. (2006) Development of a simple noninvasive index to predict significant fibrosis in patients with HIV/ HCV coinfection. Hepatology 43: 1317-1325.

24. Negredo E, Bonjoch A, Gómez-Mateu M, Estany C, Puig J, et al. (2012) Time of progression to osteopenia/osteoporosis in chronically HIV-infected patients: screening DXA scan. PLoS One 7: e46031.

25. Lawson-Ayayi S, Cazanave C, Kpozehouen A, Barthe N, Mehsen N, et al (2013) Chronic viral hepatitis is associated with low bone mineral density in HIV-infected patients, ANRS CO 3 Aquitaine Cohort. J Acquir Immune Defic Syndr 62: 430-435.

26. Lo Re V 3rd, Guaraldi G, Leonard MB, Localio AR, Lin J, et al. (2009) Vira hepatitis is associated with reduced bone mineral density in HIV-infected women but not men. AIDS 23: 2191-2198.

27. Lin JC, Hsieh TY, Wu CC, Chen PJ, Chueh TH, et al. (2012) Association between chronic hepatitis $C$ virus infection and bone mineral density. Calcif Tissue Int 91: 423-429.

28. Orsini LG, Pinheiro MM, Castro CH, Silva AE, Szejnfeld VL (2013) Bone minera density measurements, bone markers and serum vitamin $D$ concentrations in men with chronic non-cirrhotic untreated hepatitis C. PLoS One 8: e81652.

29. Milazzo L, Mazzali C, Bestetti G, Longhi E, Foschi A, et al. (2011) Liver-related factors associated with low vitamin D levels in HIV and HIV/HCV coinfected patients and comparison to general population. Curr HIV Res 9: 186-193.

30. Pludowski P, Grant WB, Bhattoa HP, Bayer M, Povoroznyuk V, et al. (2014) Vitamin d status in central europe. Int J Endocrinol 2014: 589587.

31. Holick MF, Gordon CM (2011) The Hormone Foundation's: Patient guide to vitamin D deficiency.J Clin Endocrinol Metab 96: 1-2.

32. Mansueto P, Carroccio A, Seidita A, Di Fede G, Craxì A (2013) Osteodystrophy in chronic liver diseases. Intern Emerg Med 8: 377-388.

33. Operskalski EA, Kovacs A (2011) HIV/HCV co-infection: pathogenesis, clinica complications, treatment, and new therapeutic technologies. Curr HIVIAIDS Rep 8: $12-22$.

34. Li Vecchi V, Maggi P, Rizzo M, Montalto G (2014) The metabolic syndrome and HIV infection. Curr Pharm Des 20: 4975-5003.

35. Ackert-Bicknell CL (2012) HDL cholesterol and bone mineral density: is there a genetic link? Bone 50: 525-533.

36. Wiercinska-Drapalo A, Jaroszewicz J, Tarasow E, Siergiejczyk L, Prokopowicz D (2007) The possible association between serum cholesterol concentration and decreased bone mineral density as well as intravertebral marrow fat in HIV-1 infected patients. Infection 35: 46-48. 